\title{
Time domain localization technique with sparsity constraint for imaging acoustic sources
}

\author{
Thomas Padois $^{\mathrm{a}, \mathrm{b}}$, Olivier Doutres ${ }^{\mathrm{a}}$, Franck Sgard ${ }^{\mathrm{b}}$, Alain Berry ${ }^{\mathrm{c}}$ \\ ${ }^{a}$ Department of Mechanical Engineering, École de Technologie Supérieure (ÉTS), \\ Montréal, (Qc), H3C $1 \mathrm{~K} 3$, Canada \\ ${ }^{b}$ Institut de Recherche Robert-Sauvé en Santé et en Sécurité du Travail, Montréal, (Qc), \\ H3A 3C2, Canada \\ ${ }^{c}$ Group of Acoustics of the University of Sherbrooke, Department of Mechanical \\ Engineering, Université de Sherbrooke, Sherbrooke, (Qc), J1K 2R1, Canada
}

\begin{abstract}
This paper addresses source localization technique in time domain for broadband acoustic sources. The objective is to accurately and quickly detect the position and amplitude of noise sources in workplaces in order to propose adequate noise control options and prevent workers hearing loss or safety risk. First, the generalized cross correlation associated with a spherical microphone array is used to generate an initial noise source map. Then a linear inverse problem is defined to improve this initial map. Commonly, the linear inverse problem is solved with an $l_{2}$-regularization. In this study, two sparsity constraints are used to solve the inverse problem, the orthogonal matching pursuit and the truncated Newton interior-point method. Synthetic data are used to highlight the performances of the technique. High resolution imaging is achieved for various acoustic sources configurations. Moreover, the amplitudes of the acoustic sources are correctly estimated. A comparison of computation times shows that the technique is compatible with quasi real-time generation of noise source maps. Finally, the technique is tested with real data.
\end{abstract}

Keywords: Localization, inverse method, sparsity constraint, workplace PACS: code, code

Email address: Corresponding author: Thomas.Padois@etsmtl.ca (Thomas Padois) 


\section{Introduction}

Many workers are exposed to high sound levels that may be harmful and lead to hearing loss or safety risk. Passive solutions have been developed to reduce noise emitted by acoustic sources based on acoustic panels, curtains, enclosures or damping materials. However, the first step in an acoustic diagnosis is to accurately localize the position of the noise sources in order to act at the right place. The goal of this study is to develop an acoustic tool to accurately and quickly localize acoustic noise sources and reflections.

Commonly, the dimensions of an industrial hall are large and the workers undergo the direct sound field and multiple reflections. Therefore, the source localization technique has to correctly identify all the source positions and reflections in order to adequately design and implement noise control solutions.

Acoustic intensimetry is a technique to localize noise sources [1]. The sound field around an object is scanned with a two-microphones probe in order to estimate the acoustic intensity. Then, the radiated acoustic power can be computed and can be used as input to ray tracing software to predict the sound field in a closed environment. In a workplace the noise sources are multiple and distributed, therefore it is impossible to scan all the volume. The main source positions have to be known a priori. Moreover, this technique is time consuming when the dimensions of the source are large.

An alternative technique is to use an array of microphones associated with a source localization algorithm [2]. The goal is to compensate the time or phase delay between microphones in relation to a virtual scan point. The processing is performed either in the time or frequency domain. Frequency techniques use the cross spectral matrix of the microphone signals. The most common technique is beamforming [3]. Its main disadvantage is the poor spatial resolution at low frequencies. Deconvolution techniques have been developed to improve the resolution of the noise source map $[4,5,6,7,8]$. Recent works based on inverse methods with a $l_{1}$-regularization have shown good performances $[9,12,13]$. However, in a workplace, the noise sources are generally broadband so that the computational cost is large since the processing has to be done for each frequency. Alternative strategy has been proposed based on the average of the output of beamforming obtained from different microphone array locations [14]. Despite promising results, this strategy is difficult to implement in real situations.

The most common technique in time domain is the Generalized Cross 
Correlation (GCC) method which is based on the time delay between a microphone pair [10]. This time delay can be used to generate a hyperbola for the possible source positions over the scan zone. The intersection of all the hyperbolas (for all the microphone pairs) provides the source positions.

Noël et al. [11] have used the GCC associated to an inverse problem to localize source positions in an industrial hall. The solution of the inverse problem minimizes the difference between theoretical and measured crosscorrelation functions. They obtained a noise source map with the angular energy flow received from each direction relative to the microphone array. The results are promising despite a small number of scan points and large computational cost due to the computation time of the global matrix.

The objective of this study is to propose a fast source localization technique which is able to detect the main source and reflections. Therefore, a minimization problem based on the GCC is proposed but with a different theoretical formulation and solver from Noël et al. [11]. Two different sparse representations with a $l_{1}$-norm minimization are used to solve the minimization problem. Section 2 describes the theoretical background of the proposed source localization technique. The performances of the proposed technique are compared in terms of source position detection, source level estimation and computation time with synthetic data in Section 3. Finally, the source localization technique is validated with experimental data in Section 4.

\section{Source localization technique}

\subsection{Microphone array signal}

An acoustic point source at location $\mathbf{r}_{s}$ generates a signal $s\left(\mathbf{r}_{s}, t\right)$ (with $t$ the time) recorded by a set of $M$ microphones $(m=1, . ., M)$ at location $\mathbf{r}_{m}$. Throughout the paper, bold letters denote matrices or vectors. The acoustic pressure signal $p_{m}$ recorded by a microphone $m$ in free field conditions is given by

$$
p_{m}(t)=\alpha_{m}\left(\mathbf{r}_{s}\right) s\left(\mathbf{r}_{s}, t-\Delta t_{m s}\right)+v_{m}(t),
$$

where $\alpha_{m}\left(\mathbf{r}_{s}\right)$ is the geometrical attenuation due to the propagation between the source and the microphone and $v_{m}(t)$ is an uncorrelated additive noise due to background or sensor noise. The Time of Flight (ToF) $\Delta t_{m s}$ between the source $s$ and the microphone $m$ is defined from the Euclidean distance

$$
\Delta t_{m s}=\frac{1}{c_{0}}\left\|\mathbf{r}_{m}-\mathbf{r}_{s}\right\|_{2},
$$


where $c_{0}$ is the sound velocity and $\|\cdot\|_{p}$ is the $p$-norm of a vector or matrix. The microphone array signal $y\left(\mathbf{r}_{s}, t\right)$ is given by the arithmetic mean of the microphone signals

$$
y\left(\mathbf{r}_{s}, t\right)=\frac{1}{M} \sum_{i=1}^{M} p_{i}(t) .
$$

\subsection{Time domain Beamforming}

Classically, acoustic source localization or imaging is performed using the output power of the microphone array signal $y_{e}\left(\mathbf{r}_{s}\right)$ defined for a continuous signal by

$$
y_{e}\left(\mathbf{r}_{s}\right)=\mathbf{E}\left\{y\left(\mathbf{r}_{s}, t\right)^{2}\right\}=\int_{-\infty}^{+\infty} \frac{1}{M^{2}} \sum_{i=1}^{M} \sum_{j=1}^{M} p_{i}(t) p_{j}(t) d t,
$$

where $\mathbf{E}\{\cdot\}$ is the expectation value. The output power of the microphone array signal can also be written as

$$
y_{e}\left(\mathbf{r}_{s}\right)=\frac{1}{M^{2}} \sum_{i=1}^{M} \sum_{j=1}^{M}\left(p_{i} \star p_{j}\right)(\tau),
$$

where the product $\left(p_{i} \star p_{j}\right)$ corresponds to the cross-correlation function of two microphone signals at time lag $\tau=\left(\Delta t_{j s}-\Delta t_{i s}\right)$ defined by

$$
\left(p_{i} \star p_{j}\right)(\tau)=\int_{-\infty}^{+\infty} p_{i}(t) p_{j}(t+\tau) d t .
$$

The auto-correlation terms $(i=j)$ in Eq. (6) do not bring information about the time delay estimation therefore they are not accounted for in Eq. (5). Moreover, due to the symmetry of the cross-correlation function, redundant microphone pairs are removed. Thus, if the source position is searched over a set of $\mathbf{r}_{l}$ scan points $(l=1, \ldots, L)$, the modified output power of the microphone array signal $y_{e}^{\prime}\left(\mathbf{r}_{l}\right)$ is defined by

$$
y_{e}^{\prime}\left(\mathbf{r}_{l}\right)=\frac{1}{M_{p}} \sum_{i=1}^{M} \sum_{j>i}\left(p_{i} \star p_{j}\right)\left(\Delta t_{j l}-\Delta t_{i l}\right),
$$

where $M_{p}$ is the number of microphone pairs. 


\subsection{Generalized Cross Correlation (GCC)}

To compute the cross-correlation function, the inverse Fourier of the cross-spectrum of the microphone signals $C_{i j}$ at angular frequency $\omega$ is used. A weighting function $W_{i j}(\omega)$ is introduced in the cross-correlation function which is now called Generalized Cross-Correlation (GCC) function and defined by the symbol (o),

$$
\left(p_{i} \circ p_{j}\right)(\tau)=\int_{-\infty}^{+\infty} W_{i j}(\omega) C_{i j}(\omega) \exp (j \omega \tau) d \omega,
$$

where

$$
C_{i j}(\omega)=\left(\int_{-\infty}^{+\infty} p_{i}(t) \exp (-j \omega t) d t\right)\left(\int_{-\infty}^{+\infty} p_{j}(t) \exp (-j \omega t) d t\right)^{*} .
$$

The symbol $(\cdot)^{*}$ corresponds to the complex conjugate. The weighted crossspectrum is used to accurately estimate the time delay between the microphone signals. The most common weight is the PHAse Transform (PHAT) [10] given by

$$
W_{i j}(\omega)=\frac{1}{\left|C_{i j}(\omega)\right|},
$$

where $|\cdot|$ is the absolute value. This weighting function whitens the crossspectrum of the microphone signals by normalizing it by its magnitude in order to retain the phase information only. Therefore, the modified energy of the microphone signals can now be expressed as

$$
y_{e}^{\prime}\left(\mathbf{r}_{l}\right)=\frac{1}{M_{p}} \sum_{i=1}^{M} \sum_{j>i} \int_{-\infty}^{+\infty} W_{i j}(\omega) C_{i j}(\omega) \exp \left(j \omega\left(\Delta t_{j l}-\Delta t_{i l}\right)\right) d \omega .
$$

Commonly, the set of scan points $l$ defines a surface and the result is an image coded with colors, called noise source map. In the case of a single source, the noise source map is composed of a main lobe with side and spurious lobes. The main lobe has the highest amplitude and corresponds to the source position. The side lobes are due to the finite aperture of the microphone array and spurious lobes can be considered as noise. In the case of several sources, the side lobes may overlap and create false sources and the amplitude of spurious lobes may increase and prevent the detection of sources with lower levels. Therefore, techniques which decrease the influence of side and spurious lobes have to be proposed. 


\subsection{Inverse model with sparsity constraint}

One approach to decrease the effect of side and spurious lobes and thus to improve the source localization is to define an optimization problem $J$ which consists into finding the source vector $\mathbf{x}$ (corresponding to the power of the source signal) that minimizes a cost function $\rho$ that depends on the measured noise source map $\mathbf{y}^{\prime}$ (obtained with GCC Eq. (11), where $y_{e}^{\prime}$ is an element of $\mathbf{y}^{\prime}$ ) and a modeled source map $\hat{\mathbf{y}}$

$$
J(\mathbf{x})=\min _{\mathbf{x}} \rho\left(\mathbf{y}^{\prime}, \hat{\mathbf{y}}\right) .
$$

The modeled source map is defined by the following linear system

$$
\hat{\mathbf{y}}=\mathbf{A x},
$$

where $\mathbf{A}$ corresponds to a propagation model matrix [15]. To design the propagation model matrix, an acoustic source at location $\mathbf{r}_{k}$ is considered. First the time delay estimation $\Delta t_{i j, k}$ between the source $(k)$ and a microphone pair $(i, j)$ is computed

$$
\Delta t_{i j, k}=\frac{1}{c_{0}}\left\|\mathbf{r}_{i}-\mathbf{r}_{k}\right\|_{2}-\frac{1}{c_{0}}\left\|\mathbf{r}_{j}-\mathbf{r}_{k}\right\|_{2}=\Delta t_{i k}-\Delta t_{j k} .
$$

Then the time delay estimation between a scan point at location $\mathbf{r}_{l}$ and this microphone pair $\Delta t_{i j, l}$ is computed. Finally, the difference between the time delay estimations $\left(\Delta t_{i j, k}-\Delta t_{i j, l}\right)$ is calculated. A small difference means that the scan point is potentially close to the source; conversely a large difference corresponds to a scan point far from the source. A term of the propagation matrix $\mathbf{A}$ can be defined for all the microphone pairs by

$$
a\left(\mathbf{r}_{k}, \mathbf{r}_{l}\right)=\frac{1}{M_{p}} \sum_{i=1}^{M} \sum_{j>i} \begin{cases}1 & \text { if }\left|\Delta t_{i j, k}-\Delta t_{i j, l}\right| \leq \epsilon \quad \epsilon \geq 0 \\ 0 & \text { otherwise. }\end{cases}
$$

If the difference between time delay estimations is small the value is set to 1 and 0 otherwise, this means that only the contribution of the scan point close to the source position are considered. However, since the number of sources is unknown, $\mathbf{r}_{k}$ is varied among all points of the scan area, $k=1, \ldots, L$ and the propagation matrix becomes 


$$
\mathbf{A}=\left(\begin{array}{cccc}
a\left(\mathbf{r}_{1}, \mathbf{r}_{1}\right) & a\left(\mathbf{r}_{1}, \mathbf{r}_{2}\right) & \cdots & a\left(\mathbf{r}_{1}, \mathbf{r}_{L}\right) \\
a\left(\mathbf{r}_{2}, \mathbf{r}_{1}\right) & a\left(\mathbf{r}_{2}, \mathbf{r}_{2}\right) & \cdots & a\left(\mathbf{r}_{2}, \mathbf{r}_{L}\right) \\
\vdots & \vdots & \ddots & \vdots \\
a\left(\mathbf{r}_{L}, \mathbf{r}_{1}\right) & a\left(\mathbf{r}_{L}, \mathbf{r}_{2}\right) & \cdots & a\left(\mathbf{r}_{L}, \mathbf{r}_{L}\right)
\end{array}\right)
$$

The matrix A only involves the sound speed, scan point and microphone positions and thus is independent on the sound field. The cost function $\rho$ is chosen to represent the Euclidean distance, therefore the linear least squares problem can be defined as

$$
J(\mathbf{x})=\min _{\mathbf{x}}\left\|\mathbf{y}^{\prime}-\mathbf{A} \mathbf{x}\right\|_{2}^{2} .
$$

If the number of scan points is larger than the number of sources, sparse methods can be used to solve the linear inverse problem, this involves minimizing the $l_{0}$-norm of the $\mathbf{x}$ vector. However the minimization of the $l_{0}$-norm is difficult in practice. Convex relaxation of the $l_{0}$-norm using the $l_{1}$-norm is preferred. Therefore, the linear inverse problem to be solved is

$$
J(\mathbf{x})=\min _{\mathbf{x}}\left(\left\|\mathbf{y}^{\prime}-\mathbf{A x}\right\|_{2}^{2}+\lambda\|\mathbf{x}\|_{1}\right),
$$

where $\lambda$ is a regularization parameter. In the last years, several methods have been proposed to solve linear inverse problems with sparsity constraints. In this study, the solution given by the Orthogonal Matching Pursuit (called OMP in the following) $[16,13]$ and the truncated Newton interior-point called Large Scale $l_{1}$ (called LS1 in the following) [17] are compared. Both methods have been chosen for their fast computation time.

Indeed, in the context of source localization in a workplace, the noise source maps have to be quickly generated at each workstation. Although the inverse problem to be solved is the same, each method proceeds in a different way. A theoretical comparison of the methods is out of the scope of this paper, for more information the reader is referred to a review paper on sparse representation [18]. Both methods are compared with the classical GCC in terms of source localization, sound level estimation and computational time. Each method requires user defined parameters. In OMP, the stop criterion of the iteration process is determined by the residual such as explained in Padois et al. [13], which is close to the number of sources. In LS1, the regularization parameter $\lambda$ has to be defined. Koh et al. [19] have defined a maximum regularization parameter $\lambda_{\max }$ and have suggested the 
range $\left[0.001 \lambda_{\max }: 0.1 \lambda_{\max }\right]$. Decreasing the regularization parameter value increases the computation time. Therefore, the regularization parameter chosen is $\lambda=0.1 \lambda_{\max }$. Finally, it should be noticed that the more accurate is the GCC noise source map the more efficient are the inverse methods.

\section{Numerical comparison of the source localization technique}

\subsection{Numerical set-up}

To highlight the abilities of the proposed source localization technique in perfectly controlled conditions, synthetic data are used in a free field environment. The simulated sound pressure recorded by the microphone array is computed using Eq. (1). The microphone array is designed as a compact array able to detect sources in all directions in an industrial context. It is a sphere composed of three circles as shown in Figure 1 (similar to Noël's study [11]). The radius of the larger circle is $0.25 \mathrm{~m}$ and $0.2 \mathrm{~m}$ for the smaller circles. The distance between the smaller circles and the main circle is $0.15 \mathrm{~m}$. Each circle has five microphones, therefore the total number of microphone is $M=15$. In practice, the goal is to move the microphone array at various positions in the sound field and quickly generate the corresponding noise source maps. Therefore, the microphone array needs to be compact and include few microphones in order to keep computation costs reasonable [11]. Commonly, the noise generated in industrial halls is composed of many sources and reflections over a broad frequency range. Thus the source signal considered here is a Gaussian white noise with a zero mean value and a standard deviation equal to 1 . The source level is set to $94 \mathrm{~dB}$. The acoustic time signal is sampled at 44,000 $\mathrm{Hz}$ and 16,384 points are used to compute the cross-spectrum Eq. (9). The sound speed is set to $c_{0}=340 \mathrm{~m} / \mathrm{s}$. The source locations are searched in a plane (including the source positions), called scan zone, at $1 \mathrm{~m}$ from the center of the spherical microphone array. The scan zone is a square with side equal to $1 \mathrm{~m}$. The scan zone is sampled with 21 points in each direction which leads to a total number of scan points equal to $L=441$ points and a spatial sampling of $5 \mathrm{~cm}$. The GCC Eq. (11) is computed with all the microphone pairs $M_{p}=(M \times(M-1)) / 2=105$. The PHAT weighted function is used to whiten the cross-spectrum according to Eq. (10). PHAT removes the magnitude of the cross-spectrum for all frequencies therefore the source levels can not be estimated from Eq. (11). To recover the source magnitude, a compensation factor has to be introduced in Eq. (11). The root mean square of the cross-spectrum is a scalar value and can be seen as the 
energy of the signal. Therefore, the cross-spectrum $C_{i j}$ is multiplied by its root mean square in Eq. (11). The GCC Eq. (11) is computed for all the scan points and the result obtained is called the noise source map. The center of the noise source map is at the same height than the center of the spherical microphone array (at $1 \mathrm{~m})$. The noise source map is coded with colors where darker colors correspond to louder noise sources. The dynamic range of the noise source map is $16 \mathrm{~dB}$ and $1 \mathrm{~dB}$ corresponds to one coded color.

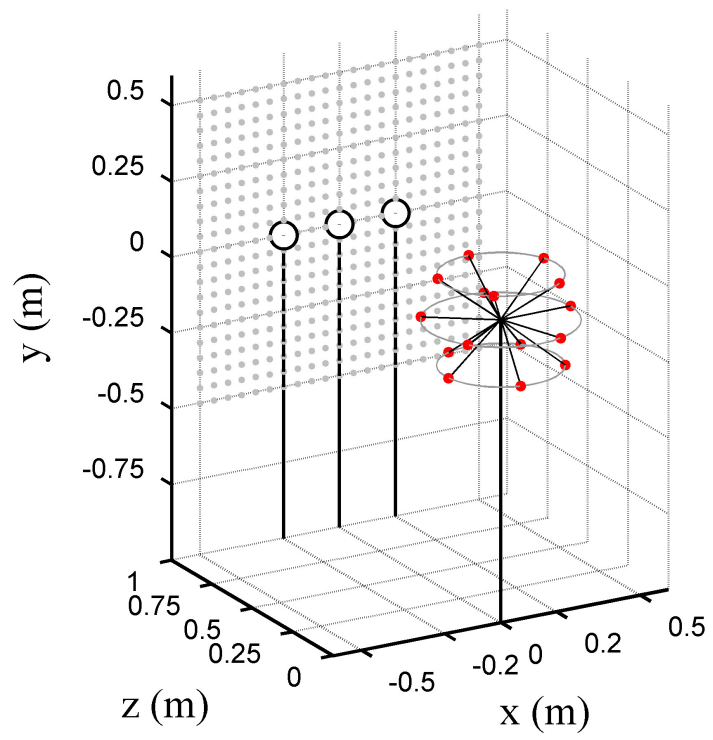

Figure 1: Spherical array composed of 15 microphones (red dots) in the case of three acoustic point sources (large circles at $z=1 \mathrm{~m}$ ). The scan zone is represented by the gray $\operatorname{dots}($ at $z=1 \mathrm{~m})$ (color online).

\subsection{Case of three uncorrelated sources}

First, the case of three uncorrelated point sources is investigated. The source spacing is $0.2 \mathrm{~m}$. The noise source maps are computed with the source localization technique discussed in Section 2 and are shown in Figure 2. The GCC noise source map exhibits three spots at the source locations with large spurious lobes. In this case, an accurate detection of the source positions is difficult. OMP and LS1 provide noise source maps with only three spots at the source locations. Both methods remove the spurious lobes and provide 
a high resolution source map. GCC and OMP correctly estimate the magnitudes of the three sources whereas LS1 under-estimates by $1 \mathrm{~dB}$ the source level. This case validates the three methods and shows the efficiency of OMP and LS1 algorithms at perfectly detecting the positions of three uncorrelated sources.

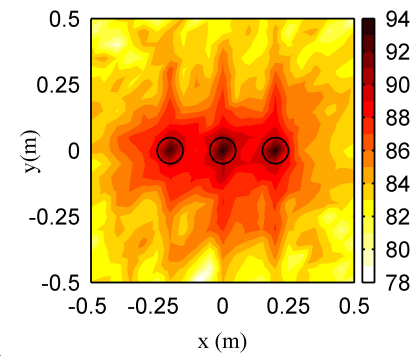

a)

b)

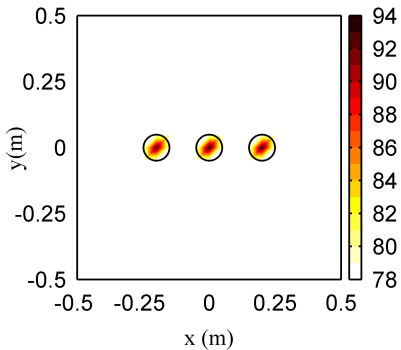

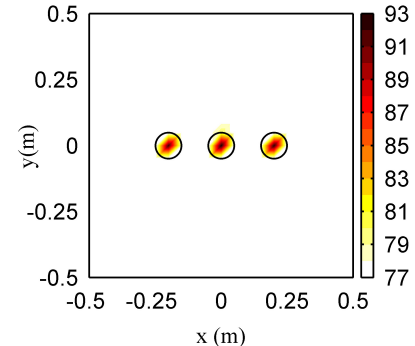

c)

Figure 2: Noise source maps for three broadband uncorrelated sources, a) GCC, b) OMP and c) LS1. The circles are the source positions. The colorbar is in $\mathrm{dB}$ (color online).

\subsection{Case of three correlated sources}

In some situations, the signals generated by sources may be correlated such as in the case of ground or wall reflections. Now, the input signal is the same for the three sources and the configuration is kept similar. The noise source maps are shown in Figure 3. GCC exhibits a main lobe at the central source position with two smaller spots at the two other source positions. In this case the pattern is clearly different from the previous configuration and it is more difficult to detect the three source positions. Due to the correlation between source signals, the side lobes merge to create a louder source at the origin. Both OMP and LS1 algorithms improve the source localization and each source is well detected. However, the source level is under-estimated for the left and right sources with each technique.

\subsection{Case of an extended source}

In the previous configuration, the noise source was a point source. However, in practical situations, noise sources are often extended. Therefore an extended source composed of 41 uncorrelated point sources from $x=-0.2 \mathrm{~m}$ to $x=0.2 \mathrm{~m}$ is computed (which means one source by centimeter). The scan 


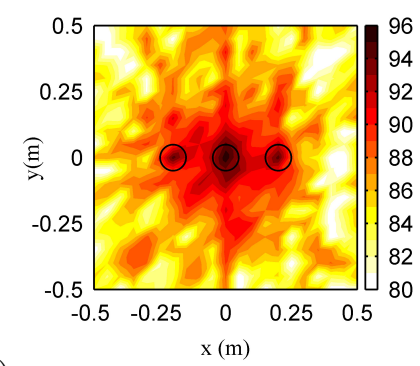

a)

b)

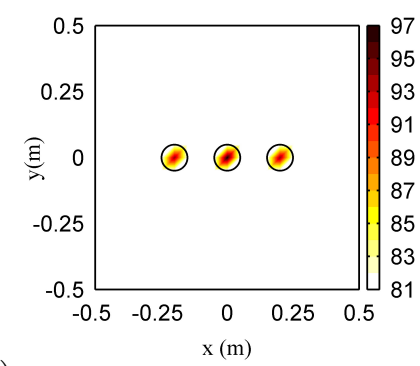

)

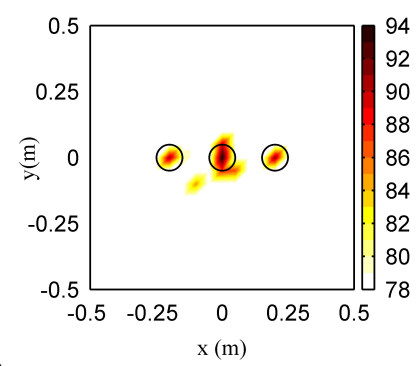

c)

Figure 3: Noise source maps for three broadband correlated sources, a) GCC, b) OMP and c) LS1. The circles are the source positions. The colorbar is in dB (color online).

zone spacing is $5 \mathrm{~cm}$ therefore the number of sources by scan point is equal to 5 , thus if the contributions of sources are summed up, an overall source level by scan point can be defined $\left(10 \log _{10}\left((5 \times 1) / 4 \times 10^{-10}\right)=101 \mathrm{~dB}\right)$. The noise source maps are shown in Figure 4. GCC shows an extended source with a large main lobe which may impair the localization of sources with a lower level. OMP and LS1 improve the noise source map. The source level estimated by both methods is close to $100 \mathrm{~dB}$. Therefore, OMP and LS1 correctly detect the source positions and moreover are able to estimate the source level with a small error.

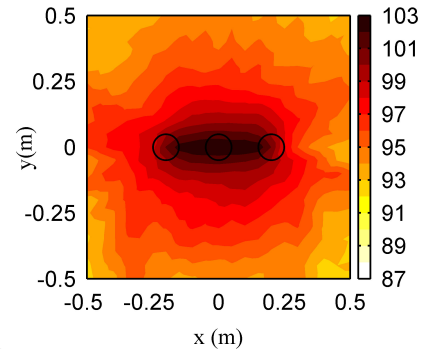

a)

b)

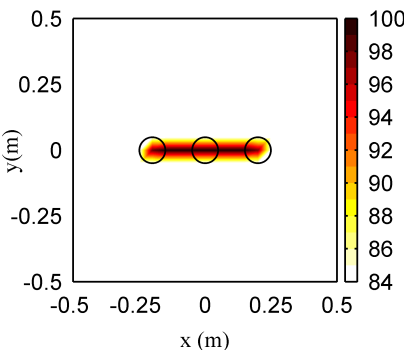

b)

Figure 4: Noise source maps for an extended source, a) GCC, b) OMP and c) LS1. The circles are the left, center and right limits of the extended source. The colorbar is in $\mathrm{dB}$ (color online). 


\subsection{Case of three sources with unequal magnitudes}

In this section, the ability to detect sources with unequal magnitudes is investigated. The configuration is similar that in Section 3.2 where three uncorrelated sources are $1 \mathrm{~m}$ from the array. However, the magnitude is decreased by $3 \mathrm{~dB}$ and $6 \mathrm{~dB}$ for the left and right sources, respectively. The noise source maps are shown in Figure 5.a-c. In each case the source positions are correctly detected but the best results are obtained with OMP and LS1. To gain insight into the noise source maps, slices at $y=0 \mathrm{~m}$ are plotted in Figure 5.d-f. These figures clearly show the high resolution ability of OMP and LS1. The best sound level estimation is given by OMP method whereas GCC and LS1 under-estimate the sound level. With LS1, it would be possible to improve the sound level estimation by decreasing the regularization parameter value.

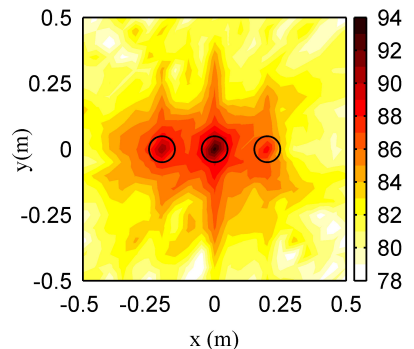

a)

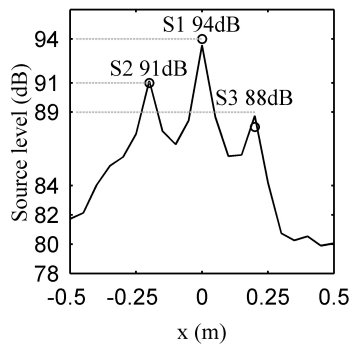

d)

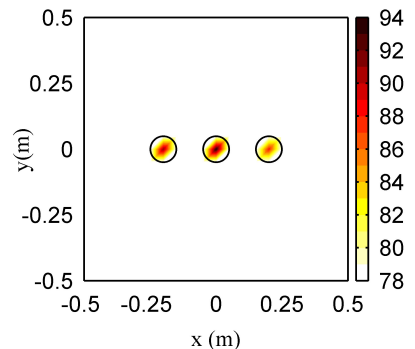

b)

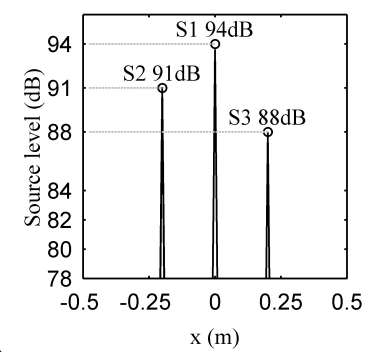

e)

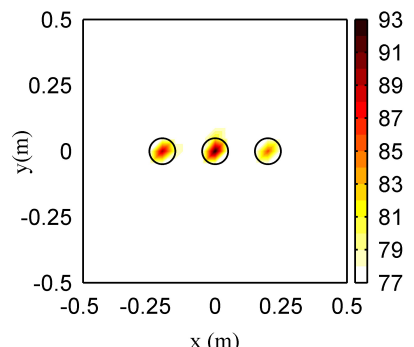

c)

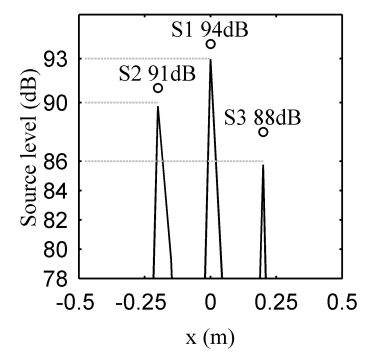

f)

Figure 5: Noise source maps for three broadband uncorrelated sources with unequal magnitudes, a) GCC, b) OMP and c) LS1 and slices at $y=0 \mathrm{~m} \mathrm{~d}$ ) GCC, e) OMP and f) LS1. The circles are the source positions and source levels. The colorbar is in $\mathrm{dB}$ (color online). 


\subsection{Computation time}

The previous sections compared the efficiency of the source localization technique to detect source positions and levels. In an industrial context, the microphone array should be moved at several positions therefore the computational time of the methods should remain reasonable. According to Noël et al. [11], their technique requires several hours with 648 scan points ( $\mathrm{PC}$ with $450 \mathrm{MHz}$ clock rate and $256 \mathrm{Mb}$ memory).

The computational time of the technique is compared for several numbers of scan points. The time is given by the tic-toc function of Matlab R2014a. A dual core processor at $3.33 \mathrm{GHz}$ is used with 4 Go of Ram. The time for building the propagation matrix A (Eq. (16)) and for solving the problem using OMP and LS1 is provided for comparison. The construction of matrix A and the implementation of OMP are custom-made codes whereas LS1 is based on the Large-Scale $l_{1}$-Regularized Least Squares Problems toolbox [19]. The computation time of GCC is very low and mainly dependent on the number of microphone pairs and is therefore not compared with the other methods. The total number of scan points ranges from $L=361$ points $(19 \times 19$ grid size $)$ up to $L=3025$ points $(55 \times 55$ grid size $)$. The result is shown in Figure 6. The time required to build matrix $\mathbf{A}$ is lower than a minute if the number of scan points is lower than 3000. Both OMP and LS1 require less than a minute if $L<3000$. Therefore, the computation time does not exceed two minutes for a scan zone with 3000 points (which is almost five times larger than Noël et al. [11]). From the trend of the curves, it is possible to define a power law depending on the number of scan points. OMP and LS1 computation times increase with the square of the number of scan points. OMP is the fastest method (for 5 iterations). Finally even with a large number of scan points, the computation time is still reasonable and can be applied at different workstations.

\section{Experimental study of the performances of the source localiza- tion technique}

\subsection{Experimental set-up}

The performances of the source localization technique have been assessed previously using synthetic data. Now, experimental data are used to confirm the previous results. Experiments were conducted in the hemi-anechoic room of the ICAR laboratory (ÉTS-IRSST, Montréal). To set-up the microphone 


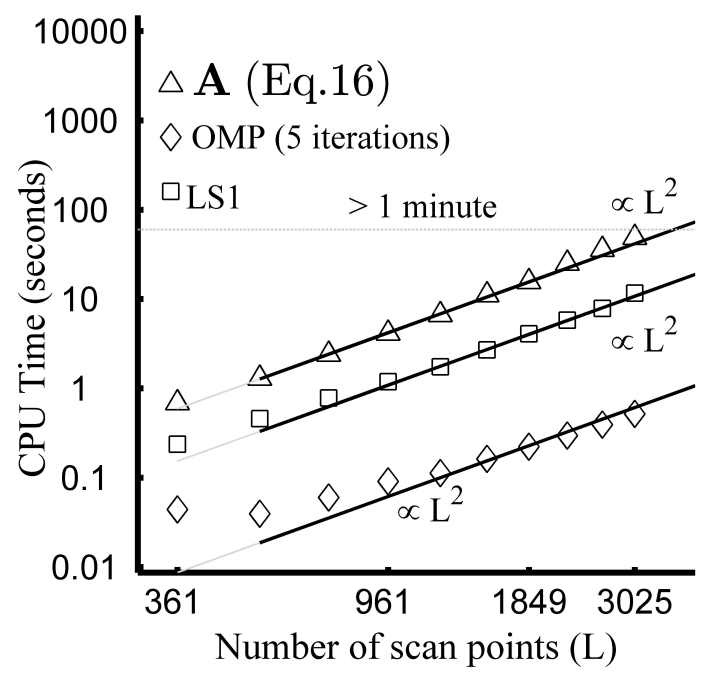

Figure 6: Computation time of OMP and LS1 versus the total number of scan points (using Matlab R2014a, running on a dual core processor at $3.33 \mathrm{GHz}$ and 4 Go of Ram).

array, a frame was composed of a sphere of radius $3.81 \mathrm{~cm}$ supported by a tripod. Holes were drilled in the sphere according to the microphone geometry. Rods with $20 \mathrm{~cm}$ length were inserted into the holes and the microphones were mounted at the end of the rods to obtain an array radius of $0.25 \mathrm{~m}$ (see Figure 7.a). Brüel\&Kjaer microphones type 4935 were used and the signals were recorded using a Brüel\&Kjaer 3038B front end and Brüel\&Kjaer Pulse software. The acoustic signals were sampled at $65,536 \mathrm{~Hz}$ during 15 seconds. The source signal was a white noise generated by a NI PXI-4461 card controlled with Labview. The signal was amplified by a BSWA audio amplifier SWA 100 and emitted by a loudspeaker. Two metal sheets were set on the ground and on the side close to the loudspeaker (see Figure 7.b). The goal is to create ground and wall reflections. The distance between the loudspeakers and the center of the microphone array was $2 \mathrm{~m}$. In this configuration, the microphone array records the direct acoustic field and the multiple reflections from the ground and walls. The scan zone where the sources are searched was a spherical grid with a radius of $2 \mathrm{~m}, \theta=[1: 360]^{\circ}$ the azimuth and $\phi=[-90: 90]^{\circ}$ the elevation. The number of scan points is 90 (respectively 45) along the azimuth (respectively elevation) which leads to a total number 
of scan points of 4050 .
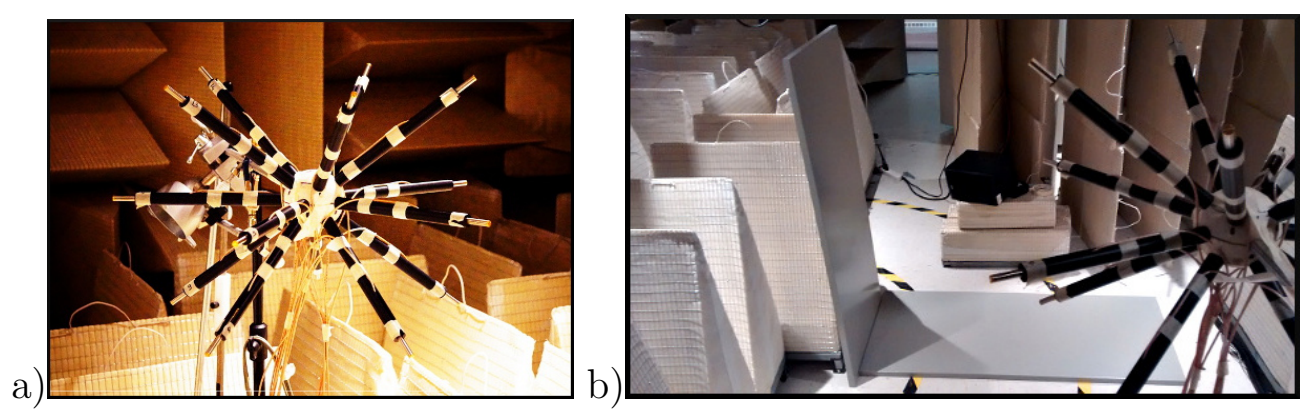

Figure 7: a) 15 spherical microphone array and b) view of the loudspeaker from the microphone array.

The noise source maps obtained with the GCC, OMP and LS1 are shown in Figure 8. The GCC noise source map exhibits several spots at the source position with a high amplitude spurious lobe. With this technique, it is really difficult to clearly identify the number of sources. OMP and LS1 methods allow for removing the spurious lobe and four main spots are detected. These sources correspond to the direct source, the ground reflection (GR), the wall reflection (WR) and the combination of both reflections $(W+G)$. Probably due to the side and spurious lobes, the GCC provides higher amplitude for the GR whereas both OMP and LS1 estimate lower amplitude. All methods find the lowest level for the $(\mathrm{W}+\mathrm{G})$ as expected. Finally the best noise source maps are provided by OMP and LS1 which allow for a clear detection of source position with a rational sound level estimation.

\section{Conclusion}

This study focuses on the source localization of acoustic sources. The objective is to quickly detect the source positions and its reflections. Three time domain source localization methods have been investigated. The Generalized Cross Correlation (GCC) provides a coarse noise source map which prevents an efficient source detection. Therefore, a linear inverse problem is defined to improve the initial map and solved with two different sparsity constraints, called Orthogonal Matching Pursuit (OMP) and truncated Newton interiorpoint (LS1). Synthetic data generated for different source configurations were used to highlight the performances of these methods. As compared to GCC, 


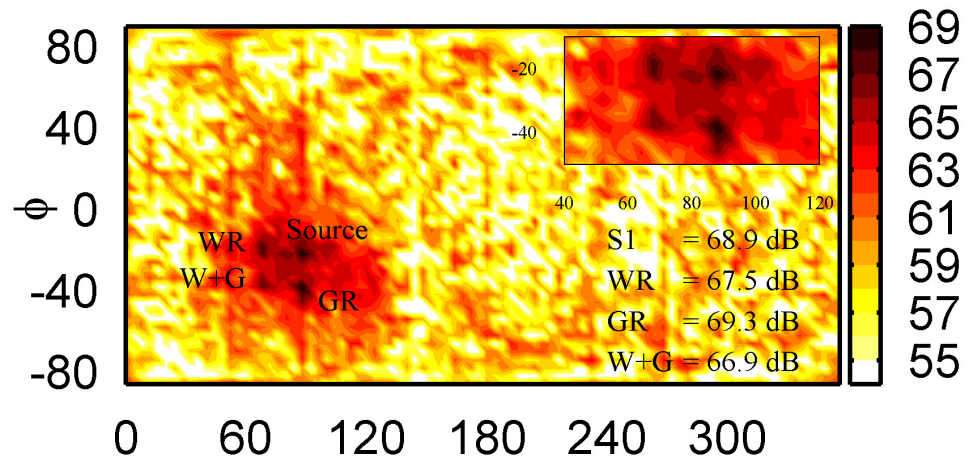

a)

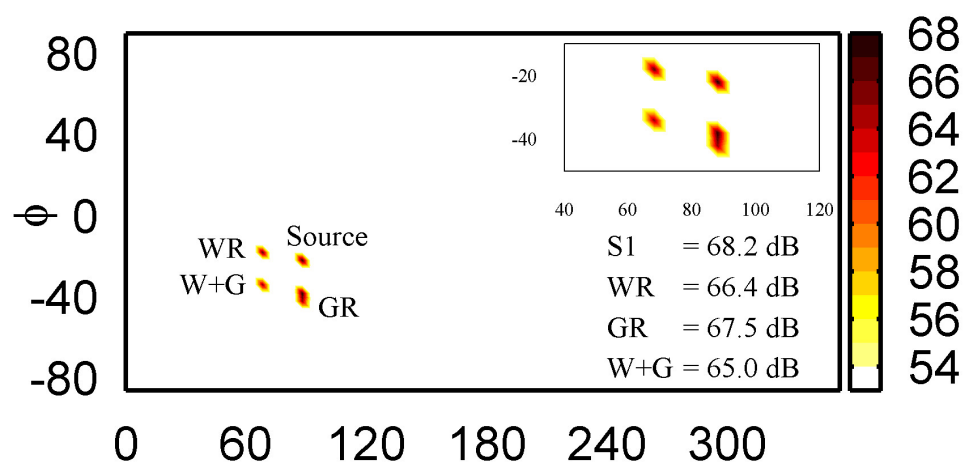

b)

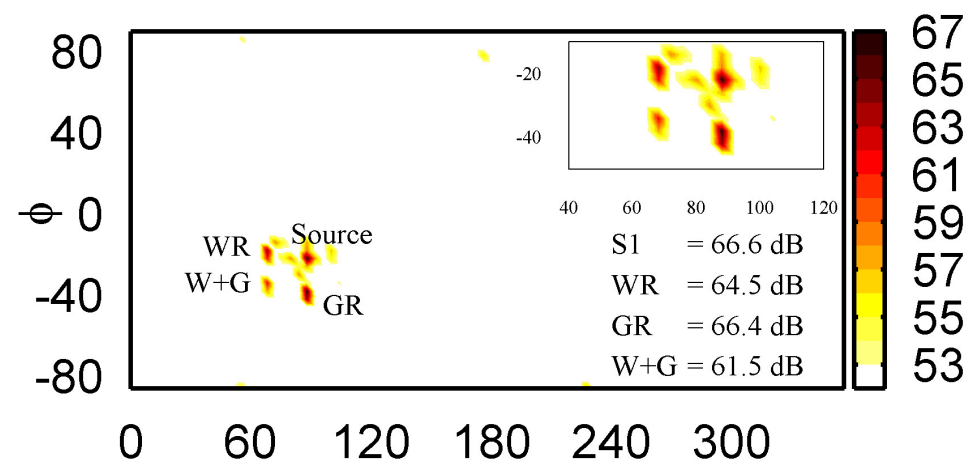

c)

$\theta$

Figure 8: Noise source maps for a loudspeakers with Ground Reflection (GR), Wall Reflection (WR) and a combination Wall-Ground (W+G), a) GCC, b) OMP and c) LS1. The colorbar is in $\mathrm{dB}$. 
sparsity constraint methods provide a high resolution imaging with a correct estimation of the source levels. Moreover, the computation time is reasonable for industrial applications. Finally an experiment has been carried out in a hemi-anechoic room that was treated to enhance wall reflections. The results have shown that both OMP and LS1 are able to localize the direct source and reflections from the ground or wall more accurately than GCC.

\section{References}

[1] J. Chatillon Influence of source directivity on noise levels in industrial halls: Simulation and experiments, Applied Acoustics, Vol.68, pages 682-698, 2007.

[2] M. Brandstein and D. Ward Microphone Arrays: Signal Processing Techniques and Applications, Springer-Verlag Berlin Heidelberg, 398 pages, 2001.

[3] U. Michel History of acoustic beamforming, $1^{\text {st }}$ Berlin Beamforming Conference (BeBeC), Berlin, Germany, 21-22 Nov, 17 pages, 2006.

[4] P. Sijtsma CLEAN based on spatial source coherence, International Journal of Aeroacoustics, Vol.6, pages 357-374, 2007.

[5] T. F. Brooks and W. M. Humphreys A deconvolution approach for the mapping of acoustic sources (DAMAS) determined from phased microphone arrays, Journal of Sound and Vibration, Vol.294, pages 856-879, 2006.

[6] T. Padois, P-A. Gauthier and A. Berry Inverse problem with beamforming regularization matrix applied to sound source localization in closed wind-tunnel using microphone array, Journal of Sound and Vibration, Vol.333, pages 6858-6868, 2014.

[7] P.A.G Zavala, W. De Roeck, K. Janssens, J.R.F Arruda, P. Sas and W. Desmet Generalized inverse beamforming with optimized regularization strategy, Mechanical Systems and Signal Processing, Vol.25(3), pages 928-939, 2011.

[8] Z. Chu and Y. Yang Comparison of deconvolution methods for the visualization of acoustic sources based on cross-spectral imaging function 
beamforming, Mechanical Systems and Signal Processing, Vol.48(1-2), pages 404-422, 2014.

[9] T. Yardibi, J. Li P. Stoica and L. N. Cattafesta III Sparsity constrained deconvolution approaches for acoustic source mapping, Journal of the Acoustical Society of America, Vol.123, pages 2631-2642, 2008.

[10] C. H. Knapp and G. C. Carter The generalized correlation method for estimation of time delay, IEEE Trans. Acoust. Speech, Signal Process., Vol.24, pages 320-327, 1976.

[11] C. Noël, V. Planeau and D. Habault A new temporal method for the identification of source directions in a reverberant hall, Journal of Sound and Vibration, Vol.296, pages 518-538, 2006.

[12] T. Padois, F. Sgard, O. Doutres and A. Berry Comparison of acoustic source localization methods in time domain using sparsity constraints, Internoise, San Francisco, USA, 9-12 August, pages 1-10, 2015.

[13] T. Padois and A. Berry Orthogonal matching pursuit applied to the deconvolution approach for the mapping of acoustic sources inverse problem, Journal of the Acoustical Society of America, Vol.138(6), pages 3678-3685, 2015.

[14] P. Castellini and A. Sassaroli Acoustic source localization in a reverberant environment by average beamforming, Mechanical Systems and Signal Processing, Vol.24, pages 796-808, 2010.

[15] J. Velasco, D. Pizarro and J. Macias-Guarasa Source localization with acoustic sensor arrays using generative model based fitting with sparse constraints, Sensors, Vol.12, pages 13781-13812, 2012.

[16] A. Peillot F. Ollivier G. Chardon and L. Daudet Localization and identification of sound using compressive sampling techniques, $18^{\text {th }}$ International Congress on Sound and Vibration (ICSV), Rio de Janeiro, Brazil, 10-14 July, 8 pages, 2011.

[17] S. Kim K. Koh M. Lustig S. Boyd and D. Gorinevsky An interior-point method for large-scale l1-regularized least squares, IEEE J. Sel. Top. Signal Process., Vol.1, pages 606-617, 2007. 
[18] Z. Zhang, Y. Xu, J. Yang, X. Li and D. Zhang A Survey of Sparse Representation: Algorithms and Applications, IEEE Access, Vol.3, pages 490-530, 2015.

[19] K. Koh S. Kim and S. Boyd A Matlab solver for large-scale l1-Regularized least squares problems, user guide, https://web.stanford.edu/ boyd/ 11_1s/11_ls_usrguide.pdf, (date last viewed 07/12/15), 6 pages, 2008. 